\title{
Syntactic Intervention cannot explain agreement attraction in English wh-questions
}

\author{
Suhas Arehalli \\ Johns Hopkins University \\ Tal Linzen \\ New York University \\ Geraldine Legendre \\ Johns Hopkins University
}

\begin{abstract}
A core goal of psycholinguistics is to account for how syntactic constraints - those that govern whether sentences in a language are grammatical or not - are enforced during real-time processing. One such constraint in Mainstream American English is subject-verb agreement, which requires the verb's number feature matches that of its subject, irrespective of the number of any other noun phrases in the sentence. Past work has demonstrated that participants often make errors when enforcing this constraint when distractor noun phrases, called attractors, have a number feature different from the subject. Franck, Lassi, Frauenfelder, and Rizzi (2006) proposes an account of agreement errors based on the position of a potential attractor in a sentence's structural derivation in modern syntactic theory. In this paper, we argue that their account predicts that we should see no agreement attraction for a particular construction in English, a Discourse-linked wh-question. In a high-powered selfpaced reading study, we test that prediction and find a robust attraction effect, suggesting that syntactic intervention is not a necessary condition for attraction errors in comprehension.
\end{abstract}

Keywords: agreement; agreement attraction; psycholinguistics; sentence processing; syntactic processing

\section{Introduction}

Human language is governed by syntactic constraints — rules that determine which sentences are considered acceptable in a given language. Syntactic constraints govern form, not meaning: they exclude ungrammatical sentences (i.e., "ideas colorless sleep green furiously") from the language, but do not necessarily exclude nonsensical ones (i.e., "Colorless green ideas sleep furiously") (Chomsky, 1965). Given the importance of syntactic constraints to language, understanding how these constraints are enforced in real-time is critical to developing a theory of sentence processing.

Much recent work in psycholinguistics has focused on characterizing the enforcement of one particular constraint: agreement. Agreement, simply put, requires that a particular syntactic feature be present on two particular words in a sentence. Mainstream American English exhibits subject-verb number agreement, where singular subjects must be found with singular verbs and vice versa: the dog runs is grammatical but the dogs runs is not. This particular constraint has been of particular interest to psycholinguists due to the existence of agreement attraction, a phenomenon where the presence of a distractor noun phrase, dubbed an attractor, results in agreement mistakenly being computed between the attractor and the verb rather than the subject and the verb (Quirk, Greenbaum, Leech, \& Svartvik, 1972; Bock \& Miller, 1991).
Agreement, and the errors caused by agreement attraction, serve as a particularly useful window into the mechanisms through which online syntactic processing occurs: On the one hand, the superficial simplicity of agreement as a syntactic constraint (merely requiring that two elements match in a particular feature) makes it an appealing phenomenon to model. On the other hand, the growing body of work investigating what factors influence the rate of attraction errors provides a complex set of behaviors that any putative model of syntactic processing must account for.

Prior work on agreement attraction has found a number of structural factors that influence the rate of attraction errors (Bock \& Cutting, 1992; Franck, Vigliocco, \& Nicol, 2002). On the basis of these results, Franck et al. (2006) argued for an account of agreement attraction tied to syntactic derivations: representations of a sentence construed as the bottomup assembly of the sentence's consituent parts. Among other things, they proposed that agreement attraction occurs as a result of syntactic intervention: It occurs only when an attractor intervenes between the two agreeing elements at the stage of the syntactic derivation in which agreement is computed, rather than in the final hierarchical or linear representation of the sentence. This account provides a tight link between the agreement configuration and how agreement is enforced during processing: the rules governing the syntactic constraint, which are encoded in the syntactic derivation, also govern the ways in which that constraint is enforced, as well as how that enforcement fails.

Franck et al. (2006)'s account also allows us to predict whether a sentence (assuming some particular syntactic analysis of that sentence) will or will not exhibit agreement attraction effects. Their evaluations of these hypotheses, conducted primarily in Italian and French, have demonstrated that many of the key predictions of this theory, including the role of intermediate states of the syntactic derivation, are consistent with experimental data. In this paper, we will evaluate the predictions of the syntactic intervention hypothesis on one particular syntactic construction, English Discourse-linked (D-linked) wh-questions ("Which trees is the gardener planting?"). We argue that a syntactic analysis with the attractor intervening on the agreement relation is unviable for D-linked wh-questions, and thus a syntactic intervention account of agreement attraction must predict that attraction should not occur in sentences of this construction. Contrary to this pre- 
diction, we note that prior evidence from offline sentence processing indicates that agreement attraction may occur (Dillon, Staub, Levy, \& Clifton Jr, 2017). Since offline judgements make it difficult to distinguish agreement errors during incremental processing from other potentially confounding effects later on, We additionally evaluate the syntactic intervention account's predictions in an online self-paced reading study, finding a robust attraction effect.

\section{Background}

Subject-verb agreement requires that the verb shares its number feature of its corresponding subject. In present tense sentences, the number feature of the verb is typically expressed in its morphology, and thus we can observe agreement failure. For instance,

\section{(1) The key is rusty.}

is grammatical, as key and is are both singular. However,

(2) The key are rusty.

is not, since the verb are is plural.

This subject-verb relationship is crucially due to their syntactic roles rather than their linear proximity. That is, the presence of any other non-subject noun phrases in the clause should not influence the verbs number. For instance,

\section{(3) The key to the cabinets are rusty.}

is still ungrammatical, despite cabinets and are both being plural. However, in both production and comprehension, speakers often mistakenly compute agreement with are and the attractor cabinets rather than with are and the subject key (Bock \& Miller, 1991, among others).

The correct subject-verb pair can be seen in the sentence's syntactic structure, the hierarchical representation posited by a syntactic formalism over which syntactic constraints can be defined. This hierarchical structure appears to be reflected in processing patterns as well: Franck et al. (2002) found that distances between the attractor and verb within these representations better predicted attraction error rates than counting the words separating the attractor and verb in the surface structure (see Fig. 1). Franck et al. (2006) propose that these representations, as well as the process of constructing these representations proposed in Minimalist approaches to syntax (Chomsky, 2000), do not only influence the strength of agreement attraction effects, but constrain when attraction errors can occur. We aim to evaluate a core claim of this proposal: that attraction occurs only when the attractor intervenes between the subject and verb at the stage of the derivation where agreement is computed.

Note that intervention at this intermediate stage is critically different than intervention in the surface structure, since the noun phrase and inflected verb can move during each step of the derivation. This can be seen most directly in cases like wh-movement. In a pair of sentences like

\section{(4) The key opens the cabinets.}

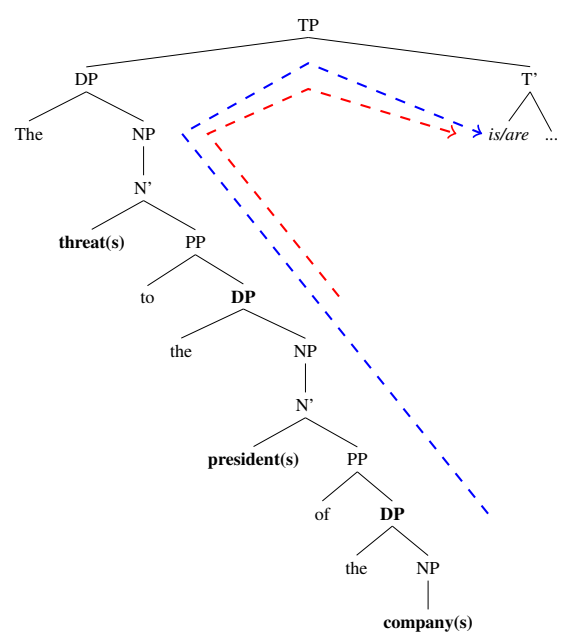

Figure 1: A simplified analysis of a stimulus from (Franck et al., 2002). While the company(s) is closer to is/are than the president $(s)$ in the sentence's linear word order, the company(s)'s distance to is in the sentence's hierarchical structure (along the blue line) is greater than that of the president(s) to $i s$. Franck et al. (2002) found a greater attraction effect emerging from the president (s) relative to the company $(s)$, indicating that hierarchically distance better predicts attraction strength than linear distance.

\section{(5) What does the key open?}

Where in example 5 the wh-word what acts as the object of the verb open, just as the cabinets does in example 4 , despite appearing at the front of the sentence rather than after open. This is typically analysed in terms of syntactic movement, where what is said to originate in the same position as the cabinets, accounting for its interpretation as the object of the sentence, but moves to its surface position at the left edge of the sentence through a series of intermediate positions to account for its secondary, quantifier-like interpretation. Franck et al. (2006)'s condition on attraction thus allows for constructions in which an attractor does not intervene in the surface structure to still elicit attraction errors as long as the attractor intervenes at the particular intermediate stage during which agreement takes place. This can be seen in the French sentence " $C$ 'est les négociations que le ministre suspend" ("It is the negotiations that the minister is suspending"), where Franck et al. (2006) argue the plural object attractor, les négociations, intervenes between the singular subject, le ministre, and the singular verb suspend, when agreement is computed before moving to its surface position before both the subject and verb (see Fig. 2).

We argue that, following a standard syntactic analysis of English, a wh-word attractor does not intervene at this intermediate stage in a particular construction called a $D$-linked wh-question. Though we lack the space to present a full exposition of the analysis we use, we provide a brief sketch of our argument for such an analysis below and attempt to con- 


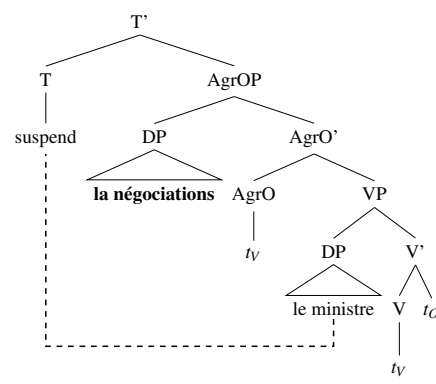

(a)

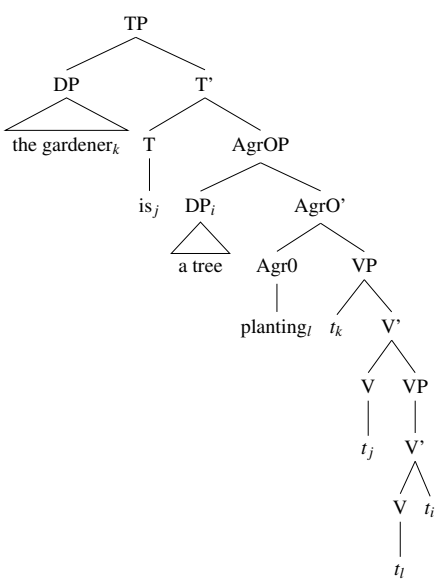

(b)

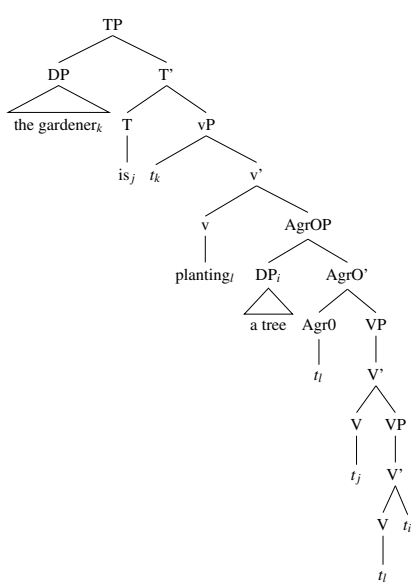

(c)

Figure 2: In the surface structure, the plural attractor les négociations appears before both the singular subject le ministre and the singular verb suspend. However, at an earlier stage of the derivation where the agreement relation is first established (the dotted line), les négociations does intervene, and thus can give rise to agreement errors under Franck et al. (2006)'s account (2a). Adopting an analysis based on that of Franck et al. (2006)'s analysis of French cleft constructions leads to failed derivation of the declarative The gardener is planting a tree. Since English lacks V to T movement for lexical verbs, the lexical verb planting remains to the right of the object due to the lack of a head position between $\mathrm{T}$ and AgrO for it to move to (2b). Our chosen analysis, which includes a vP projection, can derive the appropriate word order for the declarative form by allowing planting to move to $v(2 \mathrm{c})$.

tain the technical details to the associated figures and their captions. First, consider the following sentence, a D-linked wh-question:

\section{(6) Which trees is the gardener planting?}

Like the example in $2 \mathrm{a}$, the attractor which trees does not intervene between the subject and inflected verb in the surface structure or in the sentence's linear word order, but was generated elsewhere before moving to the left edge of the sentence. For this reason, it may be appealing to attempt to adopt a similar analysis for both constructions. However, a syntactic analysis like the one used by Franck et al. (2006) is not appropriate for our English stimuli. Our stimuli contain both an inflected auxililary and a lexical verb. While the auxiliary can move to T like in Franck et al. (2006)'s analysis, unlike French, English does not allow such movement for lexical verbs (Pollock, 1989). As a result, the lexical verb in our stimuli, planting, cannot move up to the $\mathrm{T}$ position along with are, and thus remains in a position to the right of the object. If we were to apply this analysis to the declarative counterpart of 6 (i.e., without a wh-word as the object of planting) where the object does not later move to the left edge of the sentence, we would obtain the wrong surface word order: *the gardener is a tree planting rather than the gardener is planting a tree (Fig. 2b).

We rectify this issue by adopting a more modern syntactic analysis that includes an additional projection, labeled $v \mathrm{P}$, between AgrOP and T. ${ }^{1}$ Under this analysis, the new $v P$

\footnotetext{
${ }^{1}$ Note that our word-order issue is by no means the only reason
}

projection creates a position to the left of the object for the lexical verb to move to, resulting the appropriate word order for declaratives (Fig. 2c). However, this analysis no longer leaves a position between the inflected verb is in T and subject in specvP where the attractor may intervene and cause agreement attraction.

Contrary to this prediction, Dillon et al. (2017) observe attraction effects in offline acceptability judgements, suggesting that attraction may occur despite the lack of syntactic intervention. In this paper, we adapt the materials from Dillon et al. (2017) for a high-powered self-paced reading experiment to find converging evidence for attraction in D-linked wh-questions in online, incremental processing.

\section{Methods}

\section{Participants}

320 self-identified native speakers of Mainstream American English were recruited through Prolific to participate in our experiment (Age: 18-81, mean 37.9; Gender: 176 female, 138 male, 6 other/non-binary). An additional 46 participants (age: 18-66, mean 37.6; gender: 32 female, 12 male, 1 other/non-binary, 1 chose not to identify) were recruited for a pilot study which was used for the power analysis (described in the next section) which determined the final sample size. 


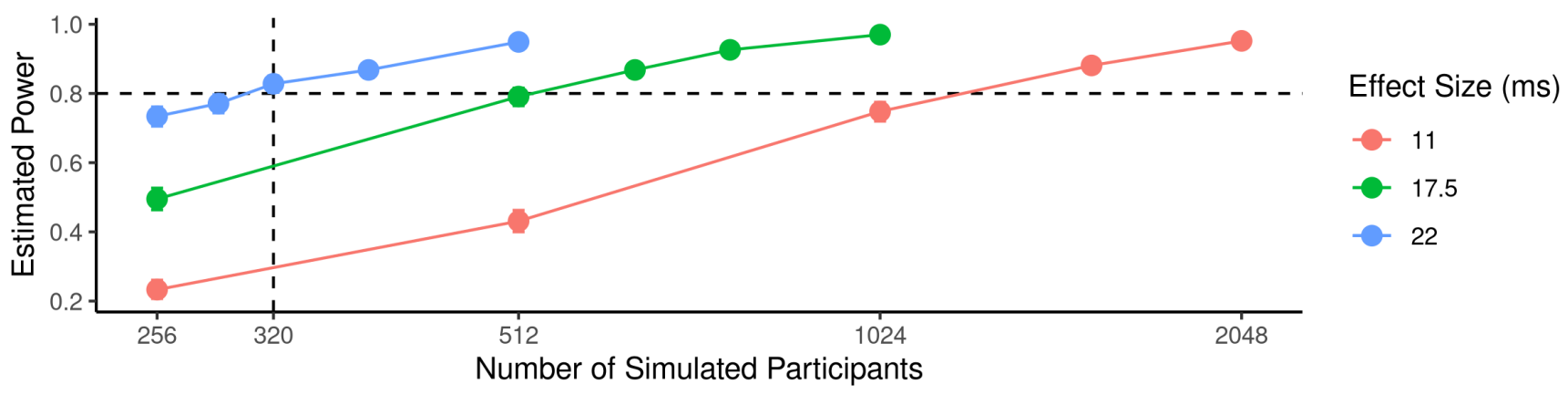

Figure 3: The power curve produced by our simulations. Error bars indicate the $95 \%$ confidence interval for the estimate. For an effect of $22 \mathrm{~ms}, 320$ participants was the smallest sample size evaluated that resulted in the $95 \%$ confidence interval lying entirely above our threshold of $80 \%$ power.

\section{Determining Power}

We model the data generation process as the output of the mixed effects model $\mathrm{rt} \sim$ match $+(1 \mid$ item $)+(1 \mid$ participant $)$. Parameter values for the random effects are estimated from our pilot, and those for the attraction effect are derived from a meta-analysis of prior self-paced reading agreement attraction studies (Jäger, Mertzen, Van Dyke, \& Vasishth, in press). In order to determine the effect of the choice of the effect size parameter, we ran the analysis three times, with the estimate of effect size being the mean effect size taken from Jäger et al. (in press), three-quarters of that value, and half of that value.

An appropriate sample size was estimated using a modified binary search of the sample size space. As our goal is to determine the minimum sample size $n$ with which we achieve a power greater than $80 \%$, we iteratively adjust our estimate of $n$ and select the smallest estimate that has such a power. In each iteration, given an estimated sample size $n_{i}$, we estimate the power of our design in 1000 simulations. If the 95\% confidence interval of this estimate lies entirely above our threshold for power (80\%), we begin the next iteration with a sample size $n_{i+1}$ halfway between $n_{i}$ and $n_{\text {lower }}$, the largest tested sample size for which the $95 \%$ confidence interval for our power estimate was not entirely above our $80 \%$ threshold. If the confidence interval for our power estimate contained or was entirely below our threshold, the next iteration began with a sample size $n_{i+1}$ halfway between $n_{i}$ and $n_{\text {upper }}$ the smallest tested sample size for which the confidence interval for the power estimate was entirely above $80 \%$. Our selection for the appropriate sample size was the value of $n_{\text {upper }}$ after 5 iterations with an initial $n_{0}$ of $256 .^{2}$

The resulting power curves are displayed in Fig. 3. Assuming an estimated agreement attraction effect of at least $22 \mathrm{~ms}$, we determined our ideal sample size was 320 participants. If we assume a smaller effect size of 17.5 or $11 \mathrm{~ms}$,

for this projection appearing here. It has been motivated by, for example, phenomena like Exceptional Case Marking, where a subject of an embedded clause is marked as an object ("I expect him to win the race").

${ }^{2}$ The full implementation of this procedure can be found with our materials at [Anonymized URL] our analysis suggests that we run 640 or 1536 participants respectively. We proceed with 320 participants, but note the limitations with respect to the effect sizes we can detect.

\section{Procedure}

Stimuli were presented to participants in a web-browser through a word-by-word self-paced moving window paradigm. At the beginning of each trial, each word in the stimulus was masked by a dashed line. By pressing the space bar, participants could have the first word of the sentence revealed. Subsequent presses of the space bar caused all of the previous words to be masked and the next word to be revealed, resulting in only a single word being revealed at any one time. Participants were instructed to read the sentence as quickly as possible while still maintaining comprehension. The time between each press of the space bar was recorded. At the end of each trial, participants were given a comprehension question that verified whether they had understood the sentence correctly.

Materials \& Design We use 16 items adapted from those of Dillon et al. (2017). Each item consists of an embedded Dlinked wh-question with the object's number either matching or mismatching the number of the subject (see Fig. 1). In order to maximize our ability to detect an attraction effect, we fix the number of the subject and the grammaticality of the sentence to values that have previously been shown to maximize the attraction effect (Ungrammatical with singular subjects: see Jäger, Engelmann, \& Vasishth, 2017). Items were modified to contain an additional prepositional phrase modifier after the critical word to ensure that any spillover effects (effects caused by a words prior to the one they are found at; ?, ?) related to agreement can be measured.

We embedded the wh-question under wonders to allow for the critical word, the verb are, to appear after both the subject, the gardener, and attractor, which tree (s). This allows us to adopt the standard interpretation of agreement attraction in self-paced reading data: Since agreement failure typically results in increased reading times at the verb, we should expect to see reading times consistent with this ungrammatical- 
Match Govinda wonders which tree the gardener are planting in the arboretum.

Mismatch Govinda wonders which trees the gardener are planting in the arboretum.

Table 1

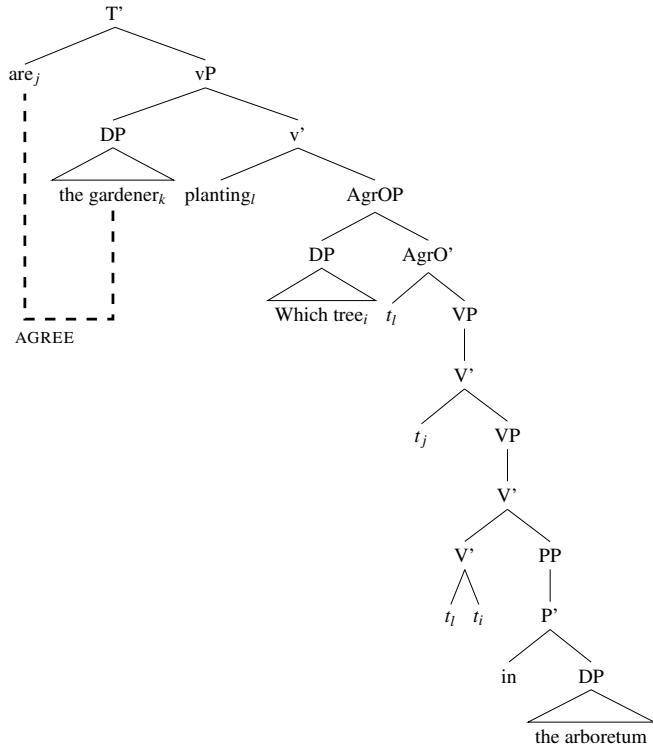

Figure 4: Our assumed analysis of the embedded clause when agreement is computed, following Pesetsky and Torrego (2001). Crucially, the attractor which tree does not intervene on the AGREE relation (i.e., is within the dashed lines).

ity at are in the MATCH condition. If no agreement attraction errors occur, the difference in the attractor's number should not influence reading times, and there will be no significant difference in reading times across conditions. However, if participants do make agreement attraction errors, we should expect those participants to compute agreement between the plural attractor and plural verb in the MISMATCH condition, resulting in lower reading times (i.e., those consistent with grammatical agreement) for those participants. As a result, we should see the mean reading time in the MismatCH condition be significantly lower than that of the MATCH condition.

\section{Analysis}

For our statistical analysis we consider the reading times at the word at which the (un)grammaticality of agreement can be determined (are, the verb) and the following two words (planting in, the spillover region) to account for potential spillover effects. Reading times associated with participants who did not achieve $80 \%$ accuracy on all comprehension questions were excluded (3.8\% data points). Additionally, outlier response times (those $>3000 \mathrm{~ms}$ ) were excluded (an additional $0.36 \%$ of all data points).

At each word, we initially analyzed the raw reading times with a linear mixed effects model, with whether the object's number matches the subject as a fixed effect and maximal random effects structure (intercepts and slopes) for items and participants. ${ }^{3}$ Factors were sum coded $+1 /-1$. If the model did not converge with a maximal random effects structure, the structure was trimmed until convergence is reached. The random effects structure that emerges from this process is reported below. These analyses were repeated with the reading times log-transformed in order to determine whether the choice of transformation affects the outcome of hypothesis testing.

As the absence of an attraction effect in these stimuli would be of particular theoretical interest, we additionally conduct a Bayesian version of these analyses. Following Schad, Nicenboim, Bürkner, Betancourt, and Vasishth (2021), we compute Bayes factors comparing models with and without an effect of the attractor's number. Priors are determined using estimates of the magnitude of the agreement attraction effect taken from (Jäger et al., 2017) and estimates of other parameters estimated from pilot data sampled from the same population of participants. Samples from the prior indicated that this parametrization reasonably approximated the distribution of self-paced reading data. As with the frequentist analyses, additional analyses over log-transformed reading times were conducted to determine whether log-transformation had an effect on the results of hypothesis testing.

\section{Results}

Reading times in our critical items are shown in Fig. 5.

\section{Frequentist Analyses}

Our analysis with raw reading times as our dependent variables showed significant effects of the attractor's number in both spillover regions (spillover 1: $\beta=-15.12,|t|=3.56$, $p<0.001$, spillover 2: $\beta=-9.26,|t|=3.31, p<0.001$ ), with a plural, mismatching attractor resulting in faster reading times. No effect was found at the verb position $(|t|=0.79$, $p=0.43)$. An analysis of log-transformed reading times showed the same pattern of results (verb: $|t|=0.036, p=$ 0.97; spillover 1: $\beta=-0.029,|t|=4.78, p<0.001$; spillover $2: \beta=-0.018,|t|=3.83, p<0.001)$.

We additionally observed an effect at the attractor position (tree (s) the gardener), outside of the critical region we planned analyses over. A post-hoc frequentist analysis over reading times at ...trees the gardener... revealed significant effects of number at trees $(\beta=7.88,|t|=2.005, p<0.05)$

\footnotetext{
${ }^{3}$ resulting in the model formula $\mathrm{rt} \sim$ match $+(1+$ match subj_id $)+(1+$ match $\mid$ item $)$
} 


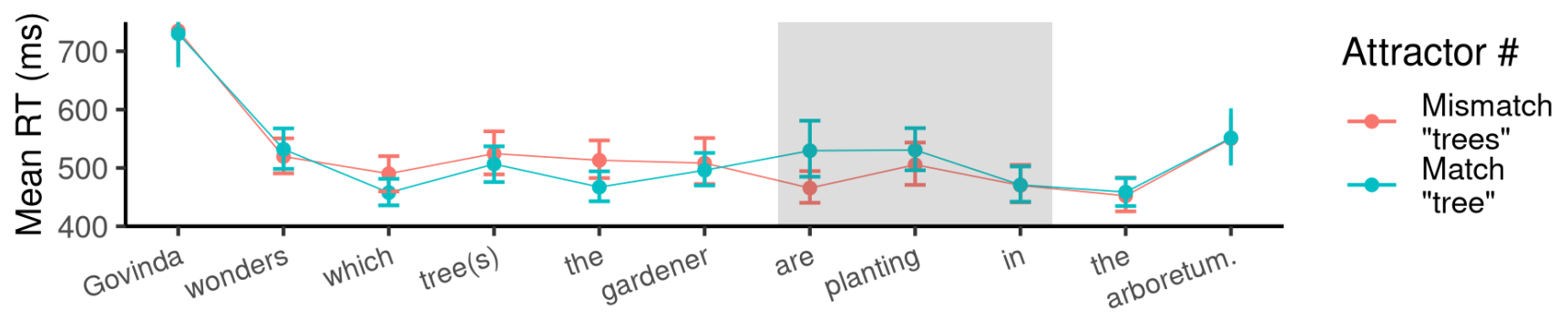

Figure 5: Word-by-word reading times from Experiment 1. Error bars indicate 95\% confidence intervals. The shaded region presents reading times for the verb and the following two words to capture spillover effects.

and the $(\beta=12.94,|t|=4.018, p<0.001)$, but not at garden $(|t|=1.45, p=0.147)$, with the plural attractor condition leading to longer reading times. As this slowdown occurs before the verb, this effect cannot be attributed to attraction. Instead, this slowdown appears to be due to a cost for processing the plural form of the attractor trees, independent of that number's effect on agreement. Since the effect is in the opposite direction of the attraction effect and appears to be non-significant by gardener, we do not believe that it presents a confound for the effect found in the critical region.

\section{Bayesian Analyses}

Analyses assuming reading times are normally distributed (equivalent to analyzing raw reading times in our frequentist models) broadly matched the findings of our frequentist analyses. We find a moderate change in evidence in favor of the alternative model (a model that assumes a difference in RTs across conditions) in the first spillover region $\left(\mathrm{BF}_{01}=0.11, \beta=-17.68, \mathrm{CI}=(-26.10,-9.55)\right)$ and the second spillover region $\left(\mathrm{BF}_{01}=0.30, \beta=-12.02, \mathrm{CI}\right.$ $=(-18.78,-5.90))$. If we assume reading times are lognormally distributed (equivalent to log-transforming reading times in our frequentist analyses), we find an extreme change in evidence in favor of the alternative model in the first spillover region $\left(\mathrm{BF}_{01}=0.0052, \beta=-0.029\right.$, $\mathrm{CI}=$ $(-0.042,-0.015))$ and a strong change in evidence in favor of the alternative model in the second spillover region $\left(\mathrm{BF}_{01}=0.073, \beta=-0.019, C I=(-0.03,-0.008)\right)$.

We conclude that the finding of attraction effects in both spillover regions is relatively robust to common variations in the analysis of self-paced reading data.

\section{Discussion}

The goal of this study was to evaluate a specific prediction made by the account of agreement attraction proposed by Franck et al. (2006): we should see no attraction in a configuration where the attractor does not intervene on the subjectverb agreement relation. We showed that an attractor does not intervene in our analysis of embedded D-linked wh-questions in Mainstream American English and in a high-powered selfpaced reading study, found evidence for a robust attraction effect. We believe that these results, along with the acceptability judgement data from Dillon et al. (2017), provide a strong case against syntactic intervention as necessary condition for attraction errors.

One possible concern with this conclusion is that we and Dillon et al. (2017) both only show agreement attraction in comprehension. The claim that agreement attraction phenomena emerge from different mechanisms in production and comprehension may allow for a version of syntactic intervention that governs agreement attraction in production, but not in comprehension. This aligns with the observation that both Franck et al. (2006)'s account and derivations in Minimalism are broadly reflective of production, where syntactic structure is build with access to a full representation of the sentence rather than incrementally. A more task-specific construal of syntactic intervention, taking into account the unique demands of the incremental processing nature of comprehension, may serve to better characterize attraction processes in tasks like self-paced reading. We see this direction as promising, calling for the evaluation of the (Franck et al., 2006)'s account's predictions with respect to our embedded D-linked wh-questions in production, as well as an investigation of the implications of a constraint like intervention on a more online comprehension-focused model of syntactic processing (i.e., Phillips, 2003; Chesi, 2012; Momma \& Phillips, 2018).

\section{Conclusion}

Prior work has found a robust, structurally sensitive effect of subject-verb agreement attraction in English: a phenomenon where speakers mistakenly compute agreement with the attractor in place of the subject. Franck et al. (2006) proposed an account of agreement attraction based on syntactic intervention, proposing that agreement attraction only occurs when the sentence's syntactic analysis has the attractor intervene on the subject-verb agreement relation at a particular stage of the derivation. We argue that for D-linked wh-questions in English, no such intervention occurs, and demonstrate a robust agreement attraction effect in self-paced reading. We conclude that a syntactic intervention account of agreement attraction in comprehension is not viable. 


\section{References}

Bock, K., \& Cutting, J. C. (1992). Regulating mental energy: Performance units in language production. Journal of Memory and Language, 31(1), 99-127. doi: 10.1016/0749596X(92)90007-K

Bock, K., \& Miller, C. A. (1991). Broken agreement. Cognitive Psychology, 23(1), 45-93.

Chesi, C. (2012). Competence and computation: toward a processing friendly minimalist grammar. UNIPRESS.

Chomsky, N. (1965). Aspects of the theory of syntax.

Chomsky, N. (2000). Minimalist inquiries: The framework. In D. M. R. Martin \& J.Uriagereka (Eds.), Step by step: Essays on minimalist syntax in honor of howard lasnik. MIT Press.

Dillon, B., Staub, A., Levy, J., \& Clifton Jr, C. (2017). Which noun phrases is the verb supposed to agree with?: Object agreement in american english. Language, 93(1), 65-96.

Franck, J., Lassi, G., Frauenfelder, U. H., \& Rizzi, L. (2006). Agreement and movement: A syntactic analysis of attraction. Cognition.

Franck, J., Vigliocco, G., \& Nicol, J. (2002). Subject-verb agreement errors in French and English: The role of syntactic hierarchy. Language and Cognitive Processes, 17(4), 371-404.

Jäger, L. A., Engelmann, F., \& Vasishth, S. (2017). Similarity-based interference in sentence comprehension: Literature review and bayesian meta-analysis. Journal of Memory and Language, 94, 316-339.

Jäger, L. A., Mertzen, D., Van Dyke, J. A., \& Vasishth, S. (in press). Interference patterns in subject-verb agreement and reflexives revisited: A large-sample study. Journal of Memory and Language.

Momma, S., \& Phillips, C. (2018). The relationship between parsing and generation. Annual Review of Linguistics, 4(1), 233-254.

Pesetsky, D., \& Torrego, E. (2001). T-to-c movement: Causes and consequences. In M. Kenstowicz (Ed.), Ken hale: a life in language. MIT Press.

Phillips, C. (2003). Linear order and constituency. Linguistic Inquiry, 34(1), 37-90.

Pollock, J.-Y. (1989). Verb movement, universal grammar, and the structure of ip. Linguistic inquiry, 20(3), 365-424.

Quirk, R., Greenbaum, S., Leech, G., \& Svartvik, J. (1972). A grammar of contemporary english. London: Longman.

Schad, D. J., Nicenboim, B., Bürkner, P.-C., Betancourt, M., \& Vasishth, S. (2021). Workflow techniques for the robust use of bayes factors. 